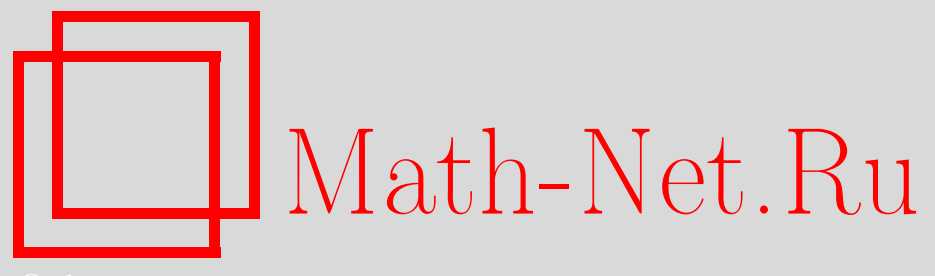

В. А. Емеличев, А. В. Пашкевич, О. А. Янушкевич, Условия эффективности решения векторной задачи дискретной оптимизации, Дискрет. матем., 1999, том 11, выпуск $1,140-145$

DOI: https://doi.org/10.4213/dm362

Использование Общероссийского математического портала Math-Net.Ru подразумевает, что вы прочитали и согласны с пользовательским соглашением http://www.mathnet.ru/rus/agreement

Параметры загрузки:

IP : 54.196 .121 .252

26 апреля 2023 г., 04:15:13

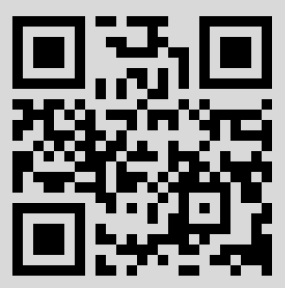




\title{
Условия эффективности решения векторной задачи дискретной оптимизации
}

(C) 1999 г. В.А. Емеличев, А.В. Пашкевич, О.А. Янушкевич

\begin{abstract}
Для некоторых классов многокритериальных задач с конечным множеством векторных оценок найдены необходимые и достаточные условия паретооптимальности решения в терминах суммы видоизмененных критериев и, в частности, в терминах суммы положительных степеней критериев.

Работа выполнена при поддержке Фонда фундаментальных исследований республики Беларусь, грант Ф97-266.
\end{abstract}

Известно, что проблема поиска всех эффективных (парето-оптимальных) решений многокритериальной (векторной) задачи оптимизации не разрешима с помощью приема линейной свертки частных критериев (см., например, $[1-5])$. Однако в случае дискретной задачи, т.е. задачи с конечным множеством векторных оценок, удалось показать [6-8], что линейная свертка неотрицательно определенных частных критериев, возведенных в некоторые положительные степени, позволяет эту проблему решить. Тем самым в указанном случае получены необходимые и достаточные условия эффективности решений векторной задачи. В этом контексте возникает вопрос, могут ли условия эффективности решения дискретной задачи быть сформулированы в терминах простейшей линейной свертки - суммы степеней частных критериев? Ниже дается положительный ответ на этот вопрос для некоторых классов многокритериальных задач с конечным множеством векторных оценок.

Пусть на множестве (допустимых) решений $X$ задана вектор-функция

$$
f=\left(f_{1}, f_{2}, \ldots, f_{n}\right): X \rightarrow \mathbf{R}^{n}, \quad n \geqslant 2 .
$$

Под $n$-критериальной задачей будем понимать задачу нахождения множества эффективных решений (множества Парето)

$$
P(X, f)=\{x \in X: \pi(x, f)=\varnothing\},
$$

где

$$
\begin{aligned}
& \pi(x, f)=\left\{x^{\prime} \in X: I\left(x, x^{\prime}\right) \cup J\left(x, x^{\prime}\right)=N_{n}, I\left(x, x^{\prime}\right) \neq \varnothing\right\} \\
& I\left(x, x^{\prime}\right)=\left\{i \in N_{n}: f_{i}(x)>f_{i}\left(x^{\prime}\right)\right\} \\
& J\left(x, x^{\prime}\right)=\left\{i \in N_{n}: f_{i}(x)=f_{i}\left(x^{\prime}\right)\right\}
\end{aligned}
$$


Введем следующие обозначения. Пусть

$$
\begin{aligned}
& \mathbf{R}_{\geqslant}^{n}=\left\{y \in \mathbf{R}^{n}: y_{i} \geqslant 0, i \in N_{n}\right\}, \\
& \mathbf{R}_{>}^{n}=\left\{y \in \mathbf{R}^{n}: y_{i}>0, i \in N_{n}\right\},
\end{aligned}
$$

и пусть $f(X)=\left\{y \in \mathbf{R}^{n}: y=f(x), x \in X\right\}$ есть множество векторных оценок.

Теорема 1. Пусть $f(X) \subset \mathbf{R}_{\geqslant}^{n},|f(X)|<\infty$. Предположим, что для $x^{0} \in X$ въполняется одно из условий: $0<f_{i}\left(x^{0}\right)<1$ для всех $i \in N_{n}$ или $f_{i}\left(x^{0}\right)>1$ для всех $i \in N_{n}$. Решение $x^{0}$ является эффективным тогда и только тогда, когда существует вектор $\varkappa \in \mathbf{R}_{>}^{n}$ такой, что для любого решения $x \in X$ справедливо неравенство

$$
\sum_{i=1}^{n} f_{i}^{\varkappa_{i}}(x) \geqslant \sum_{i=1}^{n} f_{i}^{\varkappa_{i}}\left(x^{0}\right) \text {. }
$$

Для эффективного решения $x^{0}$ в качестве $\varkappa$ можно взять любой вектор $\varkappa^{0} c$ компонентами

$$
\varkappa_{i}^{0}=\alpha \beta_{i}, \quad i \in N_{n},
$$

¿de

$$
\begin{aligned}
\alpha & \geqslant \log n / \log \min \left\{\frac{f_{i}^{\beta_{i}}(x)}{f_{j}^{\beta_{j}}\left(x^{\prime}\right)}>1: x, x^{\prime} \in X, i, j \in N_{n}\right\}, \\
\beta_{i} & =\frac{\log f_{1}\left(x^{0}\right)}{\log f_{i}\left(x^{0}\right)},
\end{aligned}
$$

$u \operatorname{moz} \partial a$

$$
\min \left\{\sum_{i=1}^{n} f_{i}^{\varkappa_{i}}(x): x \in X\right\}=n f_{1}^{\alpha}\left(x^{0}\right)
$$

Доказательство. Достаточность легко доказать методом от противного, принимая во внимание, что компоненты вектора $\varkappa$ положительны.

Докажем необходимость. Пусть $x^{0} \in P(X, f)$ и $\varkappa^{0}$ - некоторый вектор с компонентами, определяемыми формулой (2). Нетрудно видеть, что при выполнении любого из двух условий теоремы вектор $\varkappa^{0} \in \mathbf{R}_{>}^{n}$, так как числа $\alpha$ и $\beta_{i}, i \in N_{n}$, вычисляемые по формулам (3) и (4), и в том и другом случаях положительны.

Далее докажем, что при $\varkappa=\varkappa^{0}$ неравенство (1) верно для любого $x \in X$. Для этого разобьем множество $X$ на два непересекающихся подмножества

$$
\begin{aligned}
& X_{1}=\left\{x \in X: I\left(x, x^{0}\right) \cup J\left(x, x^{0}\right)=N_{n}\right\}, \\
& X_{2}=\left\{x \in X: I\left(x, x^{0}\right) \cup J\left(x, x^{0}\right) \neq N_{n}\right\} .
\end{aligned}
$$

Ясно, что для всякого решения $x \in X_{1}$ имеет место неравенство

$$
\sum_{i=1}^{n} f_{i}^{\varkappa_{i}^{0}}(x) \geqslant \sum_{i=1}^{n} f_{i}^{\varkappa_{i}^{0}}\left(x^{0}\right) .
$$


Теперь покажем, что это неравенство верно для любого $x \in X_{2}$.

Итак, пусть $x \in X_{2}$. Тогда из включения $x^{0} \in P(X, f)$ следует, что множества $I\left(x, x^{0}\right)$ и $I\left(x^{0}, x\right)$ непусты. Поэтому для любых индексов $i \in I\left(x, x^{0}\right)$ и $j \in I\left(x^{0}, x\right)$ с учетом включения $f(X) \subset \mathbf{R}_{\geqslant}^{n}$ и равенств

$$
f_{1}^{\alpha}\left(x^{0}\right)=f_{1}^{\varkappa_{1}^{0}}\left(x^{0}\right)=f_{i}^{\varkappa_{i}^{0}}\left(x^{0}\right), \quad i \in N_{n},
$$

справедливых в силу (4) и очевидных свойств чисел $\alpha$ и $\beta_{i}$

$$
\alpha=\varkappa_{1}^{0}, \quad \beta_{i} \varkappa_{1}^{0}=\varkappa_{i}^{0}, \quad i \in N_{n},
$$

выводим, что

$$
f_{i}^{\varkappa_{i}^{0}}(x)>f_{i}^{\varkappa_{i}^{0}}\left(x^{0}\right)=f_{j}^{\varkappa_{j}^{0}}\left(x^{0}\right)>f_{j}^{\varkappa_{j}^{0}}(x) .
$$

Учитывая (3) и левое неравенство в (8), находим, что

$$
\alpha \geqslant \log n / \log \delta=\log _{\delta} n,
$$

где $\delta=f_{i}^{\beta_{i}}(x) / f_{1}^{\beta_{1}}\left(x^{0}\right)$, то есть $f_{i}^{\varkappa_{i}^{0}}(x) \geqslant n f_{1}^{\varkappa_{1}^{0}}\left(x^{0}\right)$. Отсюда, принимая во внимание правое неравенство в (8), получим, что

$$
f_{i}^{\varkappa_{i}^{0}}(x)-f_{i}^{\varkappa_{i}^{0}}\left(x^{0}\right) \geqslant(n-1)\left(f_{j}^{\varkappa_{j}^{0}}\left(x^{0}\right)-f_{j}^{\varkappa_{j}^{0}}(x)\right)
$$

откуда следует, что

$$
\min \left\{f_{i}^{\varkappa_{i}^{0}}(x)-f_{i}^{\varkappa_{i}^{0}}\left(x^{0}\right): i \in I\left(x, x^{0}\right)\right\} \geqslant(n-1) \max \left\{f_{j}^{\varkappa_{j}^{0}}\left(x^{0}\right)-f_{j}^{\varkappa_{j}^{0}}(x): j \in I\left(x^{0}, x\right)\right\} .
$$

Поэтому для любого $x \in X_{2}$ верно неравенство (6), поскольку

$$
\begin{aligned}
& \sum_{i=1}^{n} f_{i}^{\varkappa_{i}^{0}}(x)-\sum_{i=1}^{n} f_{i}^{\varkappa_{i}^{0}}\left(x^{0}\right)=\sum_{i \in I\left(x, x^{0}\right)}\left(f_{i}^{\varkappa_{i}^{0}}(x)-f_{i}^{\varkappa_{i}^{0}}\left(x^{0}\right)\right)-\sum_{i \in I\left(x^{0}, x\right)}\left(f_{i}^{\varkappa_{i}^{0}}\left(x^{0}\right)-f_{i}^{\varkappa_{i}^{0}}(x)\right) \\
\geqslant & \min \left\{f_{i}^{\varkappa_{i}^{0}}(x)-f_{i}^{\varkappa_{i}^{0}}\left(x^{0}\right): i \in I\left(x, x^{0}\right)\right\}-(n-1) \max \left\{f_{i}^{\varkappa_{i}^{0}}\left(x^{0}\right)-f_{i}^{\varkappa_{i}^{0}}(x): i \in I\left(x^{0}, x\right)\right\} \geqslant 0 .
\end{aligned}
$$

Итак, неравенство (6) справедливо для любого $x \in X$, а следовательно, согласно (7) верно равенство (5). Теорема 1 доказана.

Замечание 1. Условие конечности множества векторных оценок $f(X)$ в теореме 1 необходимо для существования правой части неравенства (3).

Замечание 2. Нетрудно видеть, что строгие неравенства

$$
\left.\sum_{i=1}^{n} f_{i}^{\varkappa_{i}^{0}}(x)>\sum_{i=1}^{n} f_{i}^{\varkappa_{i}^{0}}\left(x^{0}\right)\right)
$$

для всех $x \in X \backslash\left\{x^{\prime} \in X: J\left(x^{\prime}, x^{0}\right)=N_{n}\right\}$ будут выполняться в том случае, когда неравенство (3) строгое.

Если эффективная оценка $f\left(x^{0}\right)$ не вписывается ни в одно из двух условий теоремы, то, как показывает приведенный ниже пример, утверждение теоремы 1 не верно. 
Пример 1. Пусть $n=2, X=\left\{x^{\prime}, x^{\prime \prime}\right\}, f_{1}\left(x^{\prime}\right)=3 / 4, f_{1}\left(x^{\prime \prime}\right)=1 / 2, f_{2}\left(x^{\prime}\right)=0$, $f_{2}\left(x^{\prime \prime}\right)=2$. Тогда $P(X, f)=X$.

Теперь, если предположить существование такого вектора $\left(\varkappa_{1}, \varkappa_{2}\right) \in \mathbf{R}_{>}^{2}$, что

$$
f_{1}^{\varkappa_{1}}\left(x^{\prime}\right)+f_{2}^{\varkappa_{2}}\left(x^{\prime}\right) \geqslant f_{1}^{\varkappa_{1}}\left(x^{\prime \prime}\right)+f_{2}^{\varkappa_{2}}\left(x^{\prime \prime}\right),
$$

то приходим к противоречивому неравенству

$$
\left(\frac{3}{4}\right)^{\varkappa_{1}}-\left(\frac{1}{2}\right)^{\varkappa_{1}} \geqslant 2^{\varkappa_{2}}
$$

Приведем пример, показывающий, что в утверждении теоремы 1 условие конечности множества векторных оценок $f(X)$ нельзя безоговорочно опустить.

Пример 2. Пусть $n=2, X=[-1,0], f_{1}(x)=x+3, f_{2}(x)=3-\sqrt{1-x^{2}}$.

Нетрудно видеть, что эффективное решение $x^{0}=-1$ ни при каком векторе $\varkappa=$ $\left(\varkappa_{1}, \varkappa_{2}\right) \in \mathbf{R}_{>}^{2}$ не минимизирует функцию $f_{1}^{\varkappa_{1}}(x)+f_{2}^{\varkappa_{2}}(x)$ на множестве $X$.

Действительно, пусть существует такой вектор $\varkappa$, что для всех $x \in X$

$$
f_{1}^{\varkappa_{1}}(x)+f_{2}^{\varkappa_{2}}(x) \geqslant f_{1}^{\varkappa_{1}}\left(x^{0}\right)+f_{2}^{\varkappa_{2}}\left(x^{0}\right) .
$$

Тогда для всех $x \in X \backslash\{-1\}$ должны выполняться неравенства

$$
\frac{(x+3)^{\varkappa_{1}}-2^{\varkappa_{1}}}{3^{\varkappa_{2}}-\left(3-\sqrt{1-x^{2}}\right)^{\varkappa_{2}}} \geqslant 1,
$$

которые, однако, противоречат очевидному равенству

$$
\lim _{x \rightarrow-1} \frac{(x+3)^{\varkappa_{1}}-2^{\varkappa_{1}}}{3^{\varkappa_{2}}-\left(3-\sqrt{1-x^{2}}\right)^{\varkappa_{2}}}=0 .
$$

Замечание 3. В примере 2 для эффективного решения $x^{0}=-1$ не будет существовать вектора $\varkappa \in \mathbf{R}_{>}^{2}$, удовлетворяющего неравенствам (9), даже в том случае, когда множество решений $X=[-1,0]$ заменить на следующее счетное множество

$$
\left\{0,-2^{-1}, \ldots,-\left(2^{k}-1\right) 2^{-k}, \ldots\right\} \cup\{-1\} .
$$

Введем обозначение

$$
\begin{aligned}
K(X, f, \varkappa) & =\underset{x \in \mathbf{R}_{>}^{n}}{\operatorname{argmin}}\left\{\sum_{i=1}^{n} f_{i}^{\varkappa_{i}}(x): x \in X\right\}, \\
K(X, f) & =\bigcup_{X, f, \varkappa) .}
\end{aligned}
$$

Непосредственно из теоремы 1 вытекает следующее утверждение.

Следствие 1. Пустъ для конечного множества векторных оценок $f(X)$ выполняется одно из условий

$$
\begin{aligned}
& f(X) \subset\left\{y \in \mathbf{R}^{n}: 0<y_{i}<1, i \in N_{n}\right\} \\
& f(X) \subset\left\{y \in \mathbf{R}^{n}: y_{i}>1, i \in N_{n}\right\} .
\end{aligned}
$$

Тогда имеет место равенство

$$
P(X, f)=K(X, f)
$$


Полезным обобщением теоремы 1 является следующее утверждение.

Теорема 2. Пусть $|f(X)|<\infty, x^{0} \in X$ и возрастающие функции

$$
\eta_{i}: f_{i}(X) \rightarrow \mathbf{R}_{\geqslant}, \quad i \in N_{n}
$$

подчинены условиям

$$
\eta_{1}\left(f_{1}\left(x^{0}\right)\right)=\eta_{2}\left(f_{2}\left(x^{0}\right)\right)=\ldots=\eta_{n}\left(f_{n}\left(x^{0}\right)\right)
$$

Пусть далее

$$
\alpha^{0}=\log n / \log \min \left\{\frac{\eta_{i}\left(f_{i}(x)\right)}{\eta_{j}\left(f_{j}\left(x^{\prime}\right)\right)}>1: x, x^{\prime} \in X, i, j \in N_{n}\right\} .
$$

Тогда для любого числа $\alpha \geqslant \alpha^{0}$ справедливо утверждение: $x^{0} \in P(X, f)$ тогда и толъко тогда, когда

$$
\min \left\{\sum_{i=1}^{n} \eta_{i}^{\alpha}\left(f_{i}(x)\right): x \in X\right\}=n \eta_{1}^{\alpha}\left(f_{1}\left(x^{0}\right)\right)
$$

Доказательство повторяет доказательство теоремы 1 с тем лишь отличием, что вместо положительных степеней $f_{i}^{\beta_{i}}(x), i \in N_{n}$, следует использовать возрастающие неотрицательно определенные функции $\eta_{i}\left(f_{i}(x)\right), i \in N_{n}$.

Если положить $\eta_{i}\left(f_{i}(x)\right)=f_{i}(x) / f_{i}\left(x^{0}\right), i \in N_{n}$, то из теоремы 2 легко вытекает следующее условие эффективности в терминах линейной свертки положительных степеней критериев.

Следствие 2. Предположим, что $f(X) \subset \mathbf{R}_{\geqslant}^{n},|f(X)|<\infty, x^{0} \in X, f_{i}\left(x^{0}\right)>0$, $i \in N_{n}$. Пусть

$$
\beta^{0}=\log n / \log \min \left\{\frac{f_{i}(x) f_{j}\left(x^{0}\right)}{f_{j}\left(x^{\prime}\right) f_{i}\left(x^{0}\right)}>1: x, x^{\prime} \in X, i, j \in N_{n}\right\} .
$$

Тогда для любого числа $\beta \geqslant \beta^{0}$ справедливо утверждение: $x^{0} \in P(X, f)$ тогда и толъко тогда, когда

$$
\min \left\{\sum_{i=1}^{n} \lambda_{i} f_{i}^{\beta}(x): x \in X\right\}=n
$$

əде $\lambda_{i}=f_{i}^{-\beta}\left(x^{0}\right), i \in N_{n}$.

Замечание 4. Последний результат является в некотором смысле аналогом критериев, полученных в [6-8]. Его существенное отличие (и преимущество) состоит в том, что следствием 2 не только декларируется существование чисел $\lambda_{i}, i \in N_{n}$, как это делается в [6-8], но и указываются явные формулы для их вычисления. 


\section{Список литературы}

1. Geoffrion A. M. Proper efficiency and the theory of vector maximization. J. Math. Analysis Appl. (1968) 22, №3, 618-630.

2. Подиновский В. В., Ногин В. Д. Парето-оптималъные решения многокритериалъных задач. Наука, Москва, 1982.

3. Емеличев В. А., Перепелица В. А. Сложность дискретных многокритериальных задач. Дискретная математика (1994) 6, №1, 3-33.

4. Емеличев В. А., Гладкий А. А., Янушкевич О. А. О многокритериальных задачах нахождения лексикографических оптимумов. Изв. АН Беларуси, сер. физ.-матем. наук (1996) №3, 82-86.

5. Меламед И. И. Линейная свертка критериев в многокритериальной оптимизации. $А в-$ томатика и телемеханика (1997) №9, 119-125.

6. Burkard R. E., Keiding H., Krarup J., Pruzán P. M. A relationship between optimality and efficiency in multicriteria 0-1 programming problems. Comput. Operat. Res. (1981) 8, №4, 241-247.

7. Емеличев В. А., Янушкевич О. А. Условия парето-оптимальности в дискретных векторных задачах оптимизации. Дискретная математика (1997) 9, №3, 154-160.

8. Кравцов М. К., Янушкевич О. А. О разрешимости векторной задачи с помощью алгоритма линейной свертки критериев. Матем. заметки (1997) 62, №4, 502-509.

Статья поступила 17.03.1998. 